\title{
Biaxial Ratcheting Response of SS 316 Steel
}

\author{
R.Suresh Kumar ${ }^{1, a}$, C.Lakshmana Rao ${ }^{2, b}$ and P.Chellapandi ${ }^{3, c}$ \\ ${ }^{1}$ Scientific Officer, Nuclear Engineering Group, IGCAR, Kalpakkam-603102, India \\ ${ }^{2}$ Professor, Department of Applied Mechanics, IITM, Chennai-600036, India \\ ${ }^{3}$ Associate Director, Nuclear Engineering Group, IGCAR, Kalpakkam-603102, India \\ a suresh@igcar.gov.in, ${ }^{b}$ lakshman@iitm.ac.in, ${ }^{\mathrm{c}} \mathrm{pcp} @ i g c a r . g o v . i n$.
}

\begin{abstract}
Keywords: Fast Breeder Reactor, Biaxial Ratcheting, Mechanical Behavior, Rectangular Rosette,
\end{abstract} Austenitic Stainless Steel.

\begin{abstract}
Ratcheting is one of the challenging phenomena that needs to be investigated for the Fast breeder reactor (FBRs), to arrive at the optimum structural dimensions that are safe and yet do not have undue redundancy. Austenitic stainless steel is the principal structural material for Indian FBR. Preliminary assessment indicates that there is a need to demonstrate that the main load carrying vessel made of this material can provide sufficient safety margin against ratcheting under biaxial loading conditions. This exercise calls for carrying out many simulated experiments, particularly with biaxial tension torsion specimens to generate adequate data for developing robust constitutive models to predict ratcheting. Accordingly, many biaxial tension-torsion experiments for austenitic stainless steel pipes were conducted and the best results have been reported here. The mechanical behavior of this material has been reported for a given axial tensile stress superimposed with a given range of cyclic shear stress for many cycles of loading. Rectangular rosette is used for capturing the biaxial response. Important material responses like cyclic hardening and biaxial ratcheting have been experimentally observed. Maximum accumulation of $2700 \mu$ axial strain has been observed for a loading condition of constant axial stress of $102 \mathrm{MPa}$ super imposed with a cyclic variation of shear stress amplitude of $120 \mathrm{MPa}$ over 2450 cycles. The amount of progressive accumulation of axial strain was found to be directly dependent on the number of cycles. The observed rate of axial strain accumulation found decreased with increase in number of cycles. All these results are presented in detail in this paper and important conclusions that are useful in modeling the observed behavior are discussed.
\end{abstract}

\section{Introduction}

One of the innovative features that considered for future fast breeder reactor (FBRs) is the elimination of main load carrying vessel cooling circuit. This would result in a significant economy by saving the cost of entire cooling circuit and also by reducing the main vessel diameter. However, the challenging aspect that needs to be addressed for this cost reduction, is to arrive at the structural dimensions that are safe and yet do not have undue redundancy. This requires an investigation of thermal ratcheting failure near sodium free level. Austenitic stainless steel is the principal structural material for Indian FBR structures. Preliminary assessment indicates that there is a need to demonstrate that the vessel made of this material can provide sufficient safety margin against ratcheting under biaxial loading conditions.

Mathematical modeling of the mechanical behavior of austenitic stainless steel under cyclic loading has also seen great developments during the last 30 years. However, proper constitutive models are still not available to predict ratcheting of SS316. Literature survey indicated that the material parameters derived from biaxial experiments are able to predict the material response more accurately compared to the material parameters derived from uniaxial experiments [1,2]. Hence it is necessary to carry out many simulated biaxial experiments to generate adequate data for calibrating 
constitutive models. Thus, robust constitutive models can be developed to predict ratcheting phenomenon.

Many biaxial tension-torsion experiments for austenitic stainless steel pipes were conducted and the best results have been reported in this paper. The mechanical behavior of this material is reported for a given axial tensile stress superimposed with a given range of cyclic shear stress for many cycles of loading. All those results along with some important conclusions that are useful in mathematical modeling of some of the observed behavior is also presented in detail in this paper. The experimental results are then used to assess the shakedown limit that is prescribed in the nuclear code.

\section{Biaxial Experiment}

Experimental Facility. A photograph of the biaxial testing facility is shown in Fig. 1. In this experimental facility, axial tensile as well as cyclic torsion loading can be applied to the tubular specimen. Servo hydraulic based actuators are used in the test facility to simulate static as well as dynamic loadings. Maximum axial capacity of the test facility is $20 \mathrm{kN}$ tension and that of the cyclic torsion is $\pm 100 \mathrm{Nm}$.

Biaxial Experiment. Biaxial state of stress can be simulated by various methods. Tension-torsion testing of tubular specimen is adopted in this paper. The cross coupling effect of shear and normal stress can be well captured by conducting such experiments. The purpose of this experiment was to provide sufficient data base under biaxial state

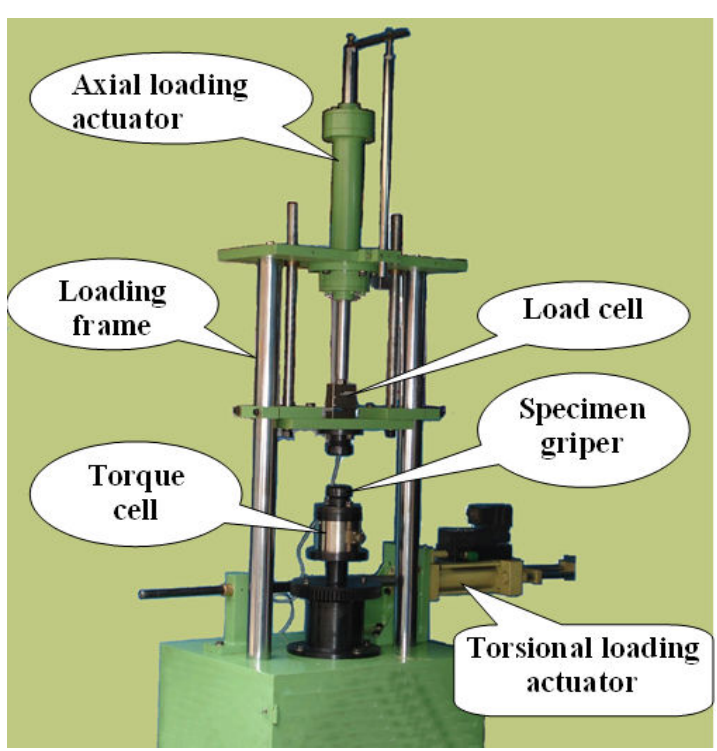

Fig. 1: Schematic of Biaxial Testing Facility of stress to establish the mechanical behavior austenitic stainless steel under biaxial fatigue loading. These data are further used for validating the mathematical models [3-4].

Specimen Preparations. Austenitic stainless steel (SS316) pipes were used in these experiments. Material properties for the SS 316 at room temperature have been taken from RCCMR 2007 [5]. Pipe dimensions are arrived at based on the loading capacity of the test facility and avoiding very thin specimens that lead to buckle of the specimen. Biaxial testing specimen has been prepared as per the guide lines of ASTM E 606-04 [6]. The pipe dimensions at the guage length portion are $18.5 \mathrm{~mm}$ OD and $0.7 \mathrm{~mm}$ thickness.

Data Acquisition. Rectangular rosette was pasted over the tubular specimen to capture axial as well as shear strains. Smallest rosette from the range available in the market was selected to get proper bondage with the cylindrical surface while applying torsion. Each terminal of the rosette was connected to separate channel data acquisition channel. The tested specimen along with the schematic loading conditions is shown in Fig. 2.

Experimental Observations. The above test facility was used for various biaxial tests by applying cyclic variation of torsion super imposed with constant axial stress. The cyclic variation of shear stress has been applied in a sinusoidal manner with a frequency of 1

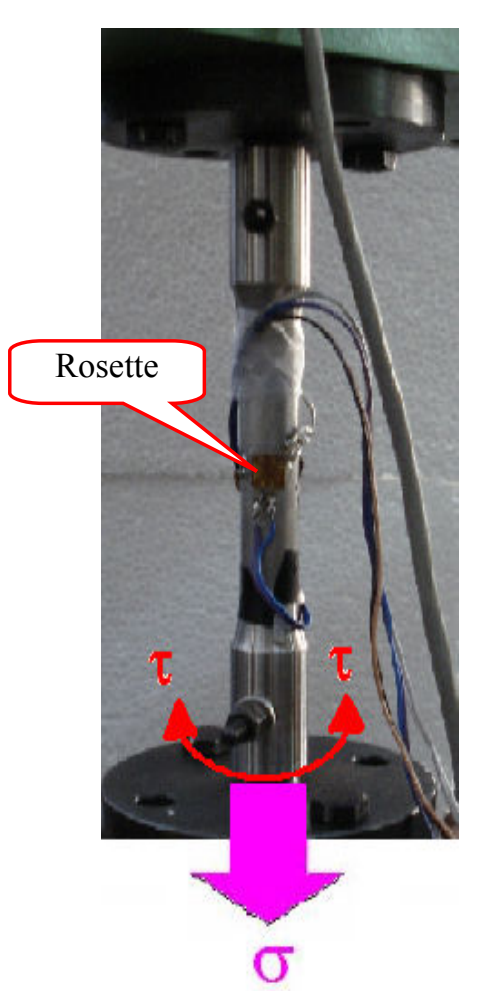

Fig. 2: Tested Specimen 
Hz. It was observed during the course of the experiment that the specimen failed by buckling and the rosette was detached from the specimen at higher torsion. Many tests were conducted and the best results are presented below:

Biaxial Tension - Torsion Test. Constant axial stress of $102 \mathrm{MPa}$ and cyclic shear stress $(\Delta \tau)$ of $120 \mathrm{MPa}$ has been applied. Some of the important results of these experiments are given in Fig 3. The biaxial ratcheting response (axial strain vs. shear strain) is shown in Fig. 3a. The axial strain is seen increasing in the direction of mean stress due to ratcheting and the mean shear strain is seen to be decreasing (the shift towards left) due to the cyclic hardening of the material. The axial stressstrain diagram is shown in Fig. 3b. For a constant stress, the strain is seen increasing in the direction of sustained stress (i.e.) ratcheting has been observed in the axial direction. The grey colour portion indicates the magnitude of the axial-shear strain during the last 200 cycles. The amount of progressive accumulation of axial strain was directly dependent on the number of cycles. The maximum accumulation of $2700 \mu$ mean axial strain has been observed for a loading condition of constant axial stress of $102 \mathrm{MPa}$ super imposed with a cyclic variation of shear stress amplitude of $120 \mathrm{MPa}$ for 2450 cycles.

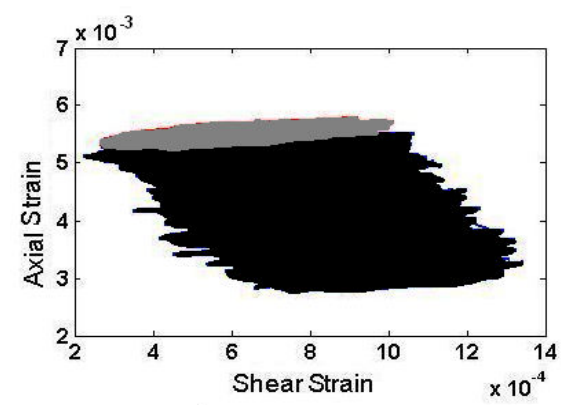

a). Biaxial Ratcheting

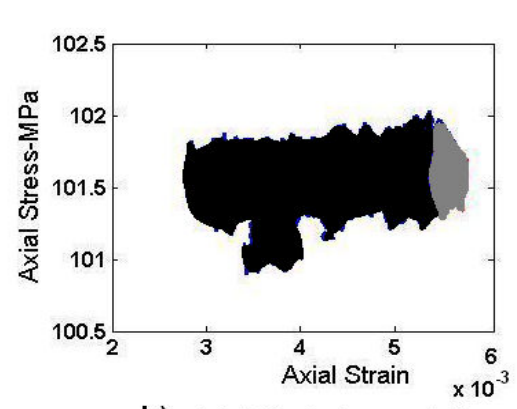

b). Axial Strain Accumulation

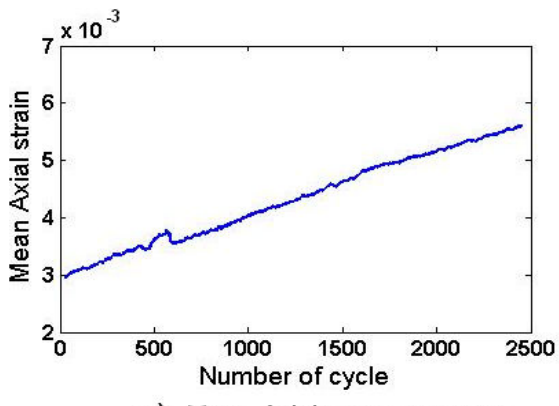

c). Mean Axial Strain Variation

Fig. 3: Biaxial Ratcheting Response

Monotonic Torsion Test. By using the above mentioned test facility monotonic torsion test was also performed. The tubular specimen was uniformly twisted and the respective torque was measured by using the torque cell attached with the specimen. Shear strain was captured using the 3 channel rectangular rosette pasted on the tubular specimen. Accordingly the shear stress shear strain diagram has been plotted for the material SS316 and it is given in Fig. 4. Thus derived shear stress -shear strain diagram can be further used for identifying material parameters for mathematically modeling the kinematic hardening behavior.

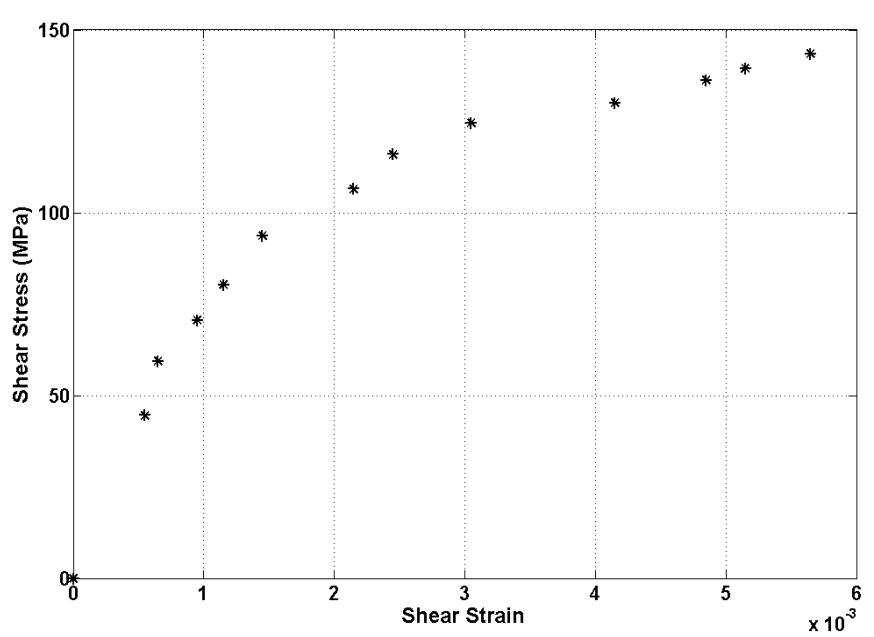

Fig. 4: Shear stress - shear strain diagram

\section{Design Code Philosophy for the Assessment of Shakedown Strain Limit.}

Design Code Procedure. RCC MR RB [7] is the design code for Indian fast breeder reactor. As per this, strain limit computation is based on the efficiency index method. Efficiency index method is used to convert the effect of biaxial stress into its equivalent uniaxial effect. Efficiency index diagram was obtained from RCC MR [7] for a given secondary stress ratio (SR). The 
secondary stress ratio (SR) is the ratio of the maximum range of cyclic normal stress to the axial normal stress. Secondary stress ratio for the loading conditions of axial stress $\left(\sigma_{\text {axial }}\right)$ of $102 \mathrm{MPa}$ and a range of cyclic shear stress of $(\Delta \tau) 120 \mathrm{MPa}$ is 1.86 . Accordingly the efficiency index obtained is 0.7 as indicated in Fig. 5a. By using this efficiency index (Fig. 5a: Courtesy from RCCMR), the biaxial loading can be converted into equivalent uniaxial load (effective stress). The shakedown strain limit for this effective stress was obtained from the uniaxial reduced cyclic curve (Fig 5b.: Obtained from the cyclic test carried out based on uniaxial specimen) is $0.07 \%$. These procedures are illustrated in Fig. 5.
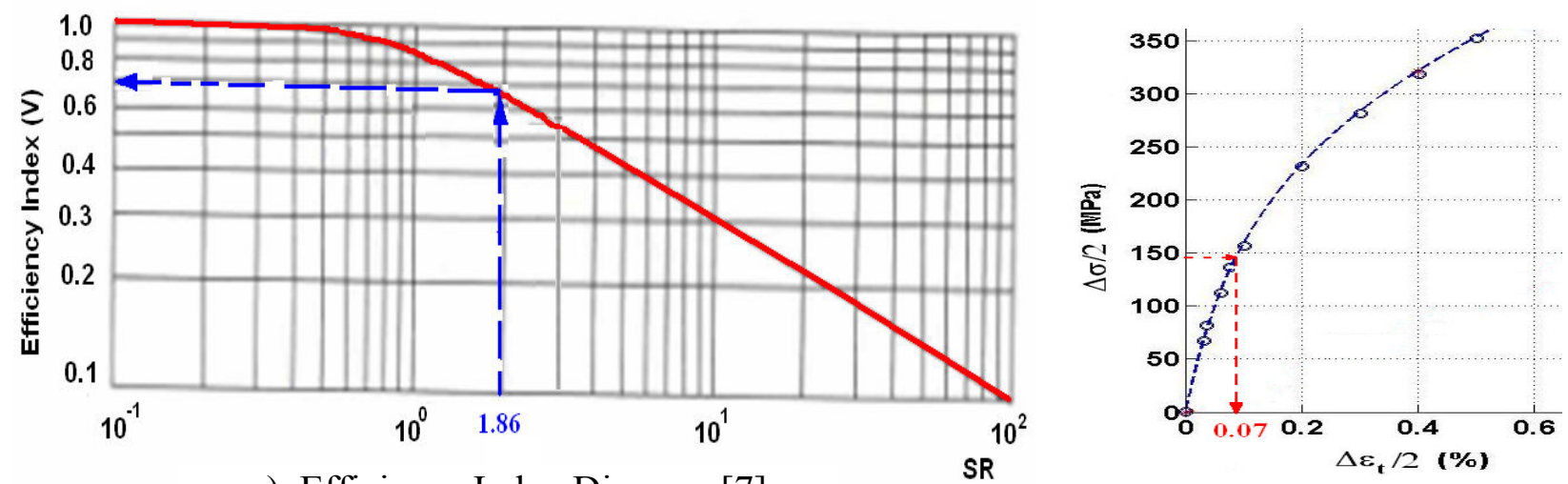

a). Efficiency Index Diagram [7]

b). Reduced Cyclic Curve

Fig 5. Shake down Strain Limit Assessment as per RCC MR Methodology

Comparison. From the above procedure, the net axial strain is obtained as $0.07 \%$. The comparative performance of the net strain computed by the efficiency index method as well as the net biaxial response obtained by experiment is shown in Fig. 6. In this figure, the bold straight line indicates the shake down limits as per the RCC-MR [7] and the wavy thin line indicates the biaxial ratcheting response obtained from the experiments. The wavy thin line shows that the experimentally obtained strain shakes down at higher number of cycles (say

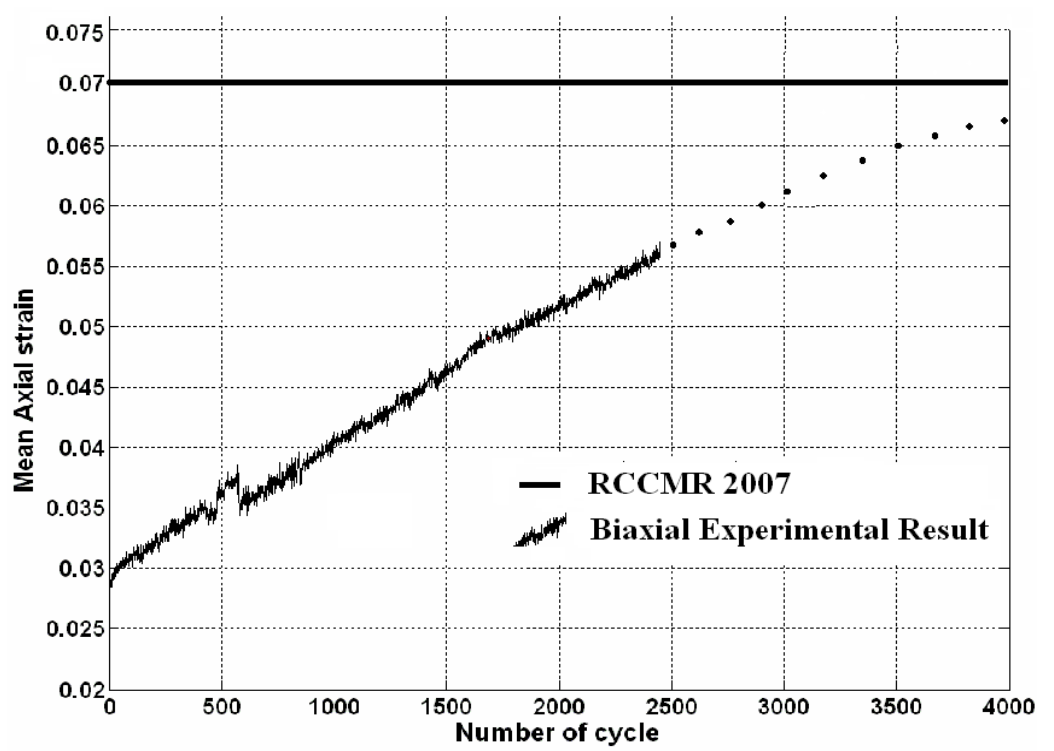

Fig. 6: Comparison of the Strain Limit around 4000). Shakedown is the situation beyond which further strain growth does not happen. Further repeating with multiple specimens will reveal the exact margin available in the RCC-MR ratcheting strain limits. The strain control cyclic testing will give more realistic shake down limits.

\section{Summary}

Experiments have been conducted to study the biaxial cyclic response of austenitic stainless steel, which is a primary material used in the FBR structures. Many biaxial tension-torsion experiments for austenitic stainless steel pipes were conducted and the best results have been reported in this 
paper. The mechanical behavior of this material is reported for a given axial tensile stress superimposed with a given range of cyclic shear stress for many cycles of loading. Biaxial ratcheting has been experimentally observed. The accumulation of $2700 \mu$ axial strain has been observed for a loading condition of constant axial stress of $102 \mathrm{MPa}$ super imposed with a cyclic variation of shear stress with a range of $120 \mathrm{MPa}$ for 2450 cycles. The amount of progressive accumulation of axial strain was observed to be dependent on the number of cycles. The decrease in slope of the mean axial strain variation (Fig. 3c.) indicated that, the rate of axial strain accumulation decreased with the increase in number of cycles. Pure torsion experiment has been conducted to generate the shear stress-shear strain curve to compute the cyclic hardening material parameters so that robust mathematical model can be developed for predicting the ratcheting phenomenon. The experimentally observed axial strain has been further compared with the shake down limit as per RCC-MR [7]. It is found that the codes predict a shake down at higher cycles (say around 4000). In order to assess the exact margin available in the codal procedure, further experiments with higher magnitude/more number of cycles have to be performed.

\section{References}

[1] Shafiqul Bari, Tasnim Hassan, in: An advancement in cyclic plasticity modeling for multiaxial ratcheting simulation, Int. J. of Plasticity 18, 873-894 (2002).

[2] M.Abdel-Karim, in: Modified kinematic hardening rules for simulations of ratcheting, Int. J. of Plasticity, Volume 25, Issue 8, Pages 1560-1587 (2009).

[3] Laurence Portiera,b, Sylvain Callocha, Didier Marquisa, Philippe Geyerb: Ratchetting under tension-torsion loadings: experiments and modeling, International Journal of Plasticity $16303-$ 335 (2000).

[4] Guozheng Kang, Qing Gao, Lixun Cai, Yafang Sun, Experimental study on uniaxial and nonproportionally multiaxial ratcheting of SS304 stainless steel at room and high temperatures, Nuclear Engg. and Design 216 13-26 (2002).

[5] RCC-MR Section I, Subsection Z, “Technical Appendix A3”, (2007).

[6] ASTM E 606-04, "Standard practice for strain controlled fatigue testing", (2005).

[7] RCC-MR Section I, Subsection B, "Design and construction rules for class-1 components of FBR nuclear islands", (2007). 